\title{
A Broadband Noise-Canceling CMOS LNA for 3.1-10.6-GHz UWB Receivers
}

\author{
Chih-Fan Liao, Student Member, IEEE, and Shen-Iuan Liu, Senior Member, IEEE
}

\begin{abstract}
An ultra-wideband 3.1-10.6-GHz low-noise amplifier employing a broadband noise-canceling technique is presented. By using the proposed circuit and design methodology, the noise from the matching device is greatly suppressed over the desired UWB band, while the noise from other devices performing noise cancellation is minimized by the systematic approach. Fabricated in a 0.18- $\mu \mathrm{m}$ CMOS process, the IC prototype achieves a power gain of $9.7 \mathrm{~dB}$ over a $-3 \mathrm{~dB}$ bandwidth of 1.2-11.9-GHz and a noise figure of 4.5-5.1 dB in the entire UWB band. It consumes $20 \mathrm{~mW}$ from a 1.8-V supply and occupies an area of only $0.59 \mathrm{~mm}^{2}$.
\end{abstract}

Index Terms-Broadband, low-noise amplifier (LNA), noise canceling, ultra-wideband (UWB).

\section{INTRODUCTION}

$\mathbf{T}$ HE DEMAND for high-speed wireless communication systems has grown during the last few years. Ultra-wideband (UWB) emerges as a new technology capable of transmitting data with low power and high data rate. It targets on applications such as wireless personal area networks (WPANs), providing seamless connectivity between consumer electronics devices for transmission of video, audio, and other high-bandwidth data [1]. Two approaches have been proposed to exploit the spectrum of 3.1-10.6-GHz allocated for UWB systems. One uses multiband OFDM modulation with 14 $528-\mathrm{MHz}$ sub-bands and a fast frequency-hopping scheme [2]. The other transmits short-duration pulses with position or polarity modulation, which results in signals spreading over several gigahertz of bandwidth [3]. Although the standard has not been completed, a front-end wideband low-noise amplifier is indispensable regardless of the receiver architecture. The amplifier must meet several stringent requirements, such as broadband input matching, sufficient gain with wide bandwidth, and low noise figure (NF), etc. In addition, it is highly desirable to implement this amplifier in CMOS technology in order to perform a high level of integration. This poses other design challenges due to the inferior RF characteristics of CMOS, such as larger parasitic capacitance, lower transconductance, and lower supply voltage.

There are several existing solutions for CMOS-based wideband amplifiers. The distributed amplifiers (DAs) provide good

Manuscript received January 22, 2006; revised August 21, 2006. This work was supported by MediaTek Inc. and the National Science Council (NSC), Taiwan.

The authors are with the Graduate Institute of Electronics Engineering and Department of Electrical Engineering, National Taiwan University, Taipei, Taiwan, 1067, R.O.C. (e-mail: 1si@cc.ee.ntu.edu.tw).

Digital Object Identifier 10.1109/JSSC.2006.889356 impedance matching, flat gain over a wide range of frequencies, and generally higher IIP3 [4], [5]. However, the demand for high-quality transmission lines makes them less attractive to low-cost applications because of the larger area. There is a 9-mW CMOS DA reported in [5] by biasing the transistors in moderate inversion. Though low power can be achieved, the cost is the lower bandwidth, i.e., 7-GHz in [5]. The resistive shunt feedback amplifiers provide wideband input matching, reducing the NF by the local feedback [6]-[8]. With sufficiently large loop gain, the NF can be brought to a low level, e.g., less than $3 \mathrm{~dB}$ in [6]. However, due to the inherently low transconductance of CMOS, large power dissipation will be required to achieve a high loop gain in a single stage [7], while stability problems will be caused with multiple stages. Recently, a new topology has been proposed for a low-power UWB LNA in a $0.18-\mu \mathrm{m}$ CMOS process [9]. It embeds the input impedance of an L-degenerated LNA into a multi-section reactive network to form a Chebyshev bandpass filter. Wideband input matching and a minimum NF of $4 \mathrm{~dB}$ are achieved with $9 \mathrm{~mW}$ power consumption. However, the NF rises rapidly at high frequencies, ranging from 5-8 dB in the bands of 7-10-GHz. This decreases the receiver sensitivity in the higher UWB bands. In addition, this topology requires a large number of high- $Q$ inductors at the input, making it difficult to realize them in a small area.

In this paper, the concept of noise cancellation [10], [11] is extended to higher frequencies by using inductive series and shunt peaking techniques and the proposed design methodology. It is shown that broadband noise cancellation effectively lowers the NF over the desired UWB band, with moderate power consumption and small die area [12]. The paper is organized as follows. Section II describes the fundamental limitations of two common wideband matching techniques, namely, common-gate and resistive shunt feedback. Analytical equations are derived to show that their performance is inadequate for a fullband UWB LNA. Section III introduces the noise-canceling technique followed by the proposed circuit topology. In Section IV, the noise analysis is performed, considering the NF at both low and high frequencies. The design methodology of this LNA is presented in Section V, in which the effect of noise cancellation and its nonidealities are discussed extensively. Experimental results for a wideband LNA implemented in a $0.18-\mu \mathrm{m}$ CMOS process are reported in Section VI. Finally, Section VII presents the conclusion.

\section{Limitations OF EXISTING WidEBAND MATCHING TECHNIQUES}

In this section, two common wideband matching techniques are reviewed in order to highlight their limitations for a fullband UWB LNA. It is assumed that the dominant noise source of a 


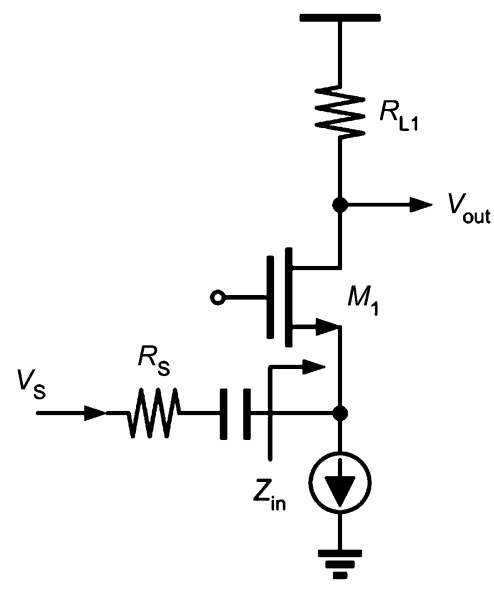

(a)

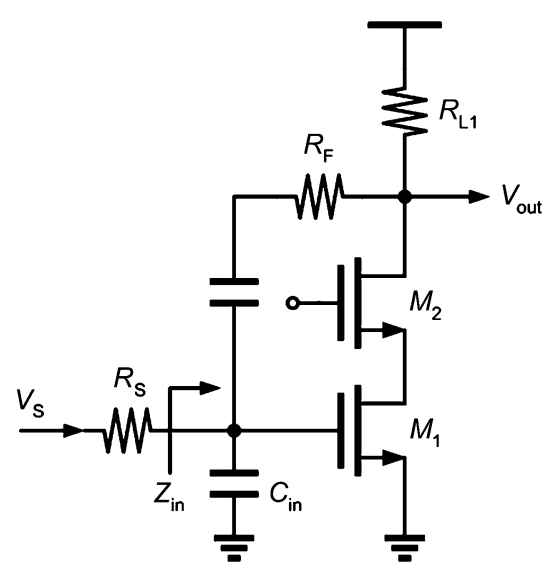

(b)

Fig. 1. Existing wideband matching techniques. (a) Common-gate. (b) Resistive shunt feedback.

MOSFET is its channel thermal noise, which has a power spectral density of $4 k T \gamma g_{\mathrm{d} 0} \Delta f$, where $g_{\mathrm{d} 0}$ is the channel conductance for $V_{\mathrm{DS}}=0$ and $\gamma$ is a noise parameter. For a deep-submicron MOSFET, the value of $\gamma$ exceeds 1 in saturation and may become $2-3$ under some biasing conditions [13]-[15].

\section{A. Common Gate}

Fig. 1(a) shows the circuit topology of a common-gate LNA. With the input impedance matched to $50 \Omega$, its noise factor $F$ $\left(\mathrm{NF}=10 \log _{10} F\right)$ is derived as

$$
F=1+\frac{\gamma}{\alpha}+\frac{4 R_{S}}{R_{L 1}}
$$

where $\alpha=g_{m} / g_{\mathrm{d} 0}$ and is often less than 1 in deep-submicron MOSFETs [13], [16]. Assuming a conservative value of 1.33 for $\gamma / \alpha$ [14], [15], the value of $R_{L 1}$ should be larger than $241 \Omega$ for a NF less than $5 \mathrm{~dB}$. With such large $R_{L 1}$, the bandwidth hardly exceeds $10 \mathrm{GHz}$ in a $0.18-\mu \mathrm{m}$ CMOS process, since the LNA must drive following circuits like I/Q downconversion mixers. ${ }^{1}$ Besides, other noise sources such as the gate-induced noise will make the NF worse. Though the capacitive cross-coupling technique [17] boosts the transconductance by a factor of 2 and makes the NF lower, the need of an external wideband balun causes additional loss in the receiver chain, compromising the overall sensitivity. Therefore, the common-gate LNA may be considered as a candidate [18] for an UWB receiver operating only in group $1(3.1-4.8 \mathrm{GHz})$, but an alternative solution will be required to enable the fullband operation with acceptable performance.

\section{B. Resistive Shunt Feedback}

The circuit topology of a resistive shunt feedback LNA is shown in Fig. 1(b). The input impedance is the parallel combination of $C_{\text {in }}$ and $\left[\left(R_{\mathrm{F}}+R_{L 1}\right) /\left(1+g_{m 1} R_{L 1}\right)\right]$, where the latter must be set to $50 \Omega$ for matching. To achieve an input reflection coefficient of $-10 \mathrm{~dB}$ at $f=10 \mathrm{GHz}$, the maximum tolerable

\footnotetext{
${ }^{1}$ According to the simulated and measured results, the bandwidth requirement while driving $M_{2}$ in Fig. 3 can be met only with $R_{L 1} \leq 180 \Omega$. Besides, driving $\mathrm{I} / \mathrm{Q}$ mixers is generally much more difficult than driving $M_{2}$, which is only 30 $\mu \mathrm{m}$ wide.
}

$C_{\text {in }}$ can be derived as $C_{\text {in }}=\left(1 / \pi R_{S} f\right) \sqrt{|\Gamma|^{2} /\left(1-|\Gamma|^{2}\right)}=$ $200 \mathrm{fF}$. Let us examine whether the constraint can be met in a $0.18-\mu \mathrm{m}$ CMOS process, with other performance being acceptable. Given a power consumption of $20 \mathrm{~mW}$ from a $1.8-\mathrm{V}$ supply, the current drawn by $M_{1}$ is $11 \mathrm{~mA}$. This combined with the constraint of $C_{\mathrm{in}}=200 \mathrm{fF}$ translate to the design of $W_{1}=100 \mu \mathrm{m}, V_{\mathrm{GS} 1}=0.85 \mathrm{~V}$, and hence $g_{m 1}=44.8 \mathrm{~mA} / \mathrm{V}$, obtained from simulation using BSIM3v3.2 model. The maximum allowable $R_{L 1}$ is primarily determined by the voltage headroom:

$$
R_{L 1} \leq \frac{V_{\mathrm{DD}}-V_{o v 1}-V_{o v 2}}{I_{D 1,2}}=\frac{1.8-0.32-0.2}{11 m}=116 \Omega
$$

where $V_{\mathrm{ov} 1}$ and $V_{\mathrm{ov} 2}$ denote the overdrive voltages of $M_{1}$ and $M_{2}$, respectively. ${ }^{2}$ The feedback resistor $R_{\mathrm{F}}$ is derived as $194 \Omega$ by $\left(R_{F}+R_{L 1}\right) /\left(1+g_{m 1} R_{L 1}\right)=50 \Omega$, while the gain is found to be $A_{V}=R_{L 1}\left(1-g_{m 1} R_{F}\right) /\left(R_{F}+R_{L 1}\right)=9.2 \mathrm{~dB}$. Neglecting the noise of the cascode device, $M_{2}$, the noise factor $F$ can be derived as

$$
\begin{aligned}
F=1+\frac{R_{F}}{R_{S}}\left(\frac{1+g_{m 1} R_{S}}{1-g_{m 1} R_{F}}\right)^{2} & +\frac{1}{R_{S} R_{L 1}}\left(\frac{R_{F}+R_{S}}{1-g_{m 1} R_{F}}\right)^{2} \\
& +\frac{\gamma g_{m 1}}{\alpha R_{S}}\left(\frac{R_{F}+R_{S}}{1-g_{m 1} R_{F}}\right)^{2}
\end{aligned}
$$

The NF is found to be $4.9 \mathrm{~dB}$ by substituting the design parameters into (3) and again assuming $\gamma / \alpha=1.33$. As the frequency increases, the noise from $M_{2}$ is more pronounced, making the NF larger than $5 \mathrm{~dB}$ for frequencies beyond several gigahertz. Though inductive peaking can be applied to the input to tolerate a larger size of $M_{1}$ and hence a higher $g_{m 1}$, this topology typically requires larger power consumption [7] or more advanced technologies [8] to achieve an acceptable NF. This is primarily due to the inherently low transconductance of CMOS, which not only degrades the noise performance but also prohibits the use of a large feedback resistor. These limitations necessitate some

${ }^{2}$ With a smaller $V_{\mathrm{ov} 2}, R_{L 1}$ in (2) can be larger to increase the loop gain and to decrease the NF, but the capacitance of $M_{2}$ will degrade the bandwidth at the cascode node. 


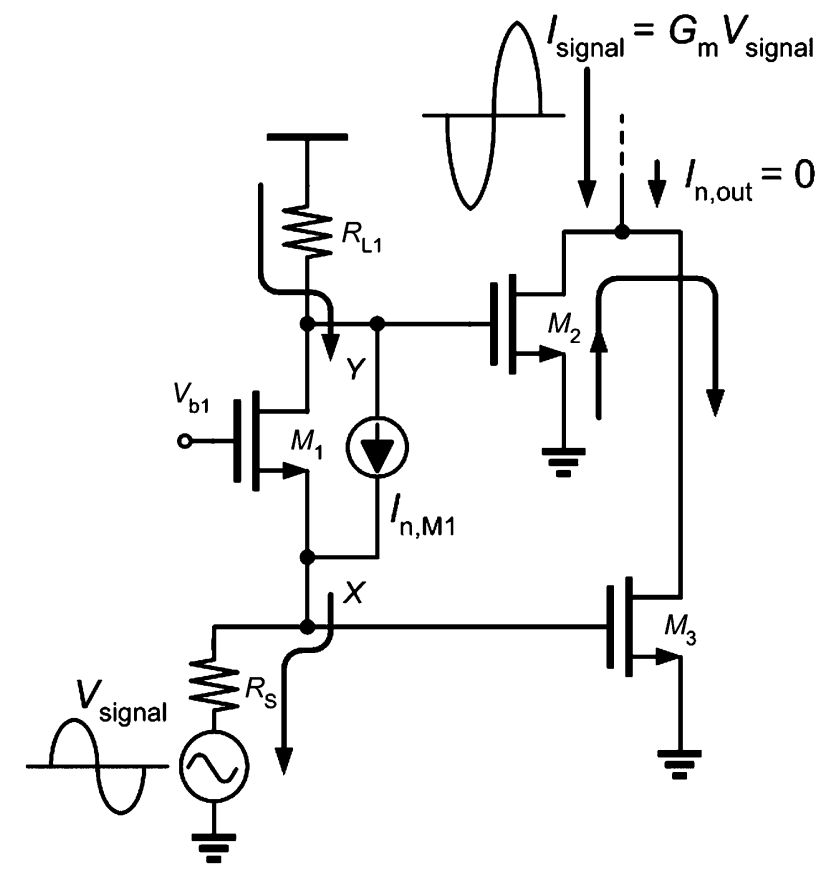

Fig. 2. Principle of the noise-canceling technique.

other wideband low-noise matching techniques for a fullband UWB LNA.

\section{BROADBAND NOISE-CANCELING LNA}

In this section, the principle of noise cancellation is presented first, followed by the proposed circuit topology of the LNA.

\section{A. Noise-Canceling Principle}

The purpose of noise cancellation is to decouple the input matching with the NF by canceling the output noise from the matching device [10], [11]. Fig. 2 illustrates an example, which is based on a common-gate LNA. The input matching is accomplished by setting $1 / g_{m 1}$ to $50 \Omega$. The noise current of $M_{1}$, which is modeled by the current source $I_{n, M 1}$, flows into node $X$ but out of node $Y$. This creates two fully correlated noise voltages at nodes $X$ and $Y$ with opposite phases. These two voltages are converted to currents by $M_{3}$ and $M_{2}$, respectively. By properly designing $g_{m 3}$ and $g_{m 2}$, the noise contributed by $M_{1}$ can be cancelled at the output. On the other hand, the signal voltages at nodes $X$ and $Y$ are in phase, resulting in constructive addition at the output. The condition for complete noise cancellation is derived as

$$
\begin{aligned}
& I_{n, \mathrm{out}}=\frac{I_{n, M 1}}{1+g_{m 1} R_{S}} R_{L 1} g_{m 2}-\frac{I_{n, M 1}}{1+g_{m 1} R_{S}} R_{S} g_{m 3}=0 \\
& \Rightarrow g_{m 2} R_{L 1}=g_{m 3} R_{S}
\end{aligned}
$$

while the equivalent transconductance, $G_{m}$, is found to be

$$
G_{m}=\frac{I_{\text {signal }}}{V_{\text {signal }}}=\frac{1 / g_{m 1}}{R_{S}+1 / g_{m 1}}\left(g_{m 1} R_{L 1} g_{m 2}+g_{m 3}\right) .
$$

Compared to resistive shunt feedback amplifiers, the noise factor contributed by the matching device is now fully decoupled with the input matching condition. Note that $M_{2}$ can be replaced with a pMOS transistor to reuse the bias current of $M_{3}$ if the resultant bandwidth is acceptable. The variable-gain method in [18] can also be applied here, since both circuits are based on a common-gate topology. On the contrary, it is difficult to vary the gain of a feedback amplifier without affecting other performance.

\section{B. Proposed LNA Circuit Topology}

The proposed broadband noise-canceling LNA is depicted in Fig. 3 [12]. The gate bias of $M_{3}$ is given by an off-chip bias-T, which also provides the DC current path of $M_{1}$. A current source is used to bias $M_{2}$, and a $4 \mathrm{pF}$ bypass capacitor provides an ac ground over the desired band. Inductors $L_{1}$ and $L_{2}$ are used for shunt peaking, extending the bandwidth efficiently without any high- $Q$ requirement [19]. The series inductor $L_{3}$ further resonates with the input capacitance of $M_{4}$, resulting in a larger bandwidth and some residual peaking on the frequency response [16], [20]. Practical $Q$ factors of on-chip inductors limit the peaking to be less than $1 \mathrm{~dB}$. It is therefore highly desirable to implement inductors $L_{1}, L_{2}$, and $L_{3}$ as narrow traces to save the area, without sacrificing the bandwidth or the NF. Compared to [9], which requires various high- $Q$ inductors at the input, the chip area of our proposed circuit can be greatly reduced. To facilitate testing, an output matching stage is included. Note that the output $50 \Omega$ matching is not required in an integrated receiver, hence the load resistor, $R_{L 3}$, can be directly replaced with a switching quad to form a single-balanced mixer. Typical loading of I/Q mixers on an LNA is thus considered here. The performance of this circuit is primarily determined by the design of the first two stages. In the following section, the noise analysis is performed, followed by an optimization procedure for this LNA.

\section{NOISE ANALYSIS}

By applying the noise-canceling technique, the NF of the proposed LNA is dominated by $R_{L 1}, M_{2}$, and $M_{3}$. At low frequencies, assuming that (4) and the input matching condition, $R_{S}=1 / g_{m 1}$, are satisfied, the noise factors contributed by $R_{L 1}, M_{2}$, and $M_{3}$ can be derived as

$$
\begin{aligned}
F_{R L 1} & =\frac{4 k T R_{L 1} g_{m 2}^{2}}{k T R_{S}\left(g_{m 3}+g_{m 2} R_{L 1} / R_{S}\right)^{2}}=\frac{R_{S}}{R_{L 1}} \\
F_{M 2} & =\frac{4 k T g_{m 2} \gamma / \alpha}{k T R_{S}\left(g_{m 3}+g_{m 2} R_{L 1} / R_{S}\right)^{2}}=\frac{R_{S}}{R_{L 1}} \frac{\gamma}{\alpha} \frac{1}{g_{m 2} R_{L 1}} \\
F_{M 3} & =\frac{4 k T g_{m 3} \gamma / \alpha}{k T R_{S}\left(g_{m 3}+g_{m 2} R_{L 1} / R_{S}\right)^{2}}=\frac{\gamma}{\alpha} \frac{1}{g_{m 3} R_{S}} .
\end{aligned}
$$

Thus, the total noise factor $F$ is approximated as

$$
F=1+\frac{R_{S}}{R_{L 1}}+\frac{R_{S}}{R_{L 1}} \frac{\gamma}{\alpha} \frac{1}{g_{m 2} R_{L 1}}+\frac{\gamma}{\alpha} \frac{1}{g_{m 3} R_{S}} .
$$

Equation (9) provides design insights for sizing circuit components, which will be discussed in depth in the next section. Note that another benefit of noise cancellation is that it lowers $F_{R L 1}$ by a factor of 4 , which is seen by comparing (6) with (1). For high-bandwidth applications, in which the large $R_{L 1}$ is not allowed, the noise-canceling topology reduces the noise from $R_{L 1}$ considerably. 


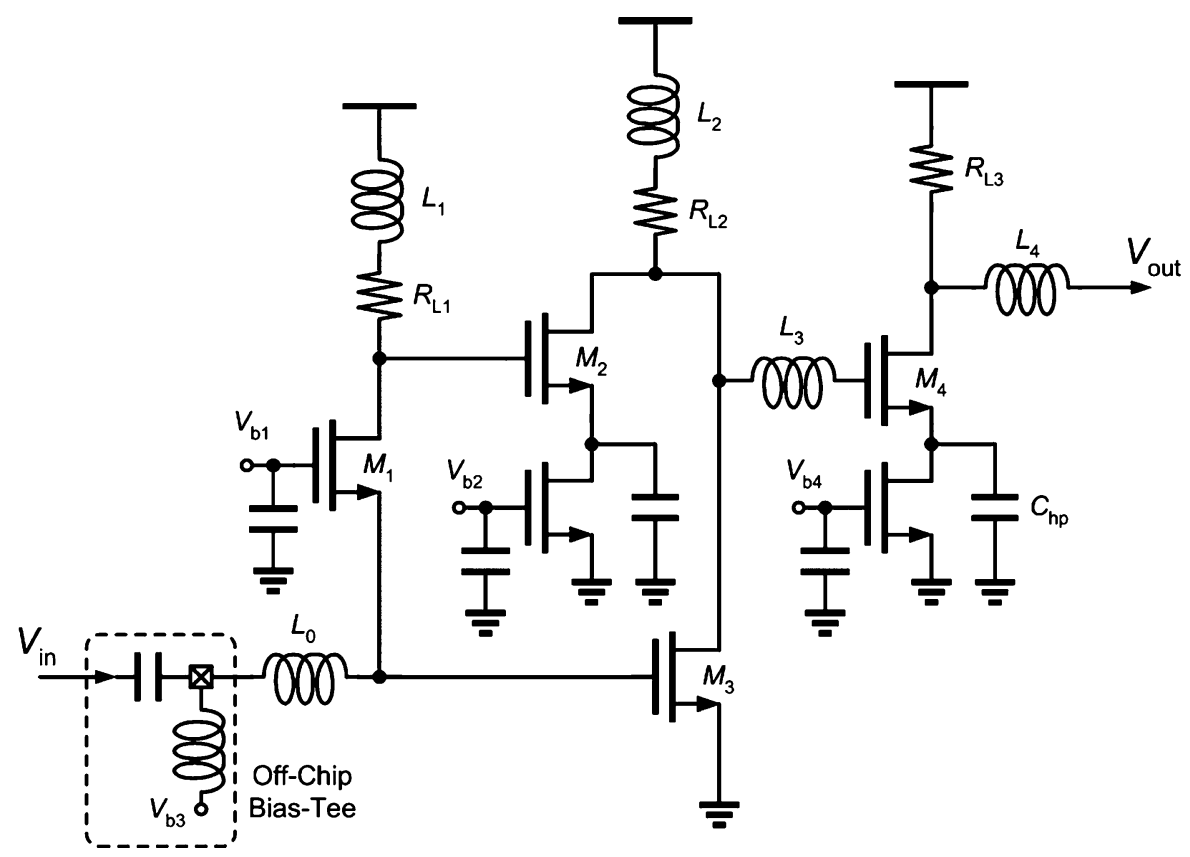

Fig. 3. Circuit topology of the proposed broadband noise-canceling LNA.

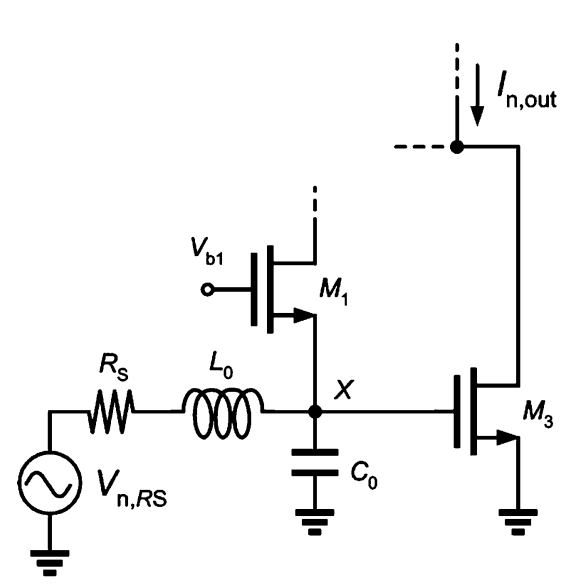

(a)

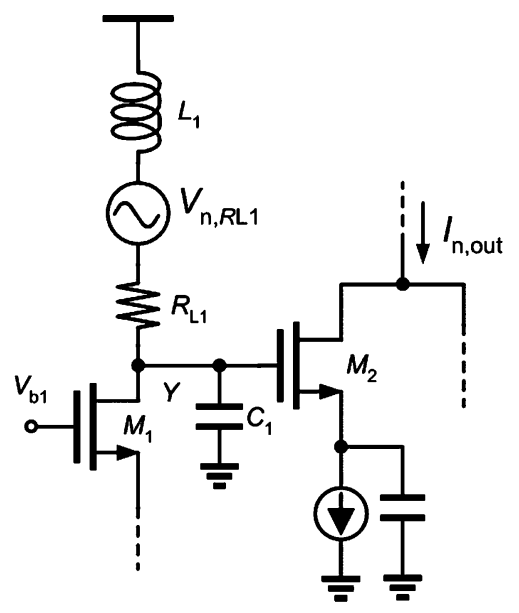

(b)

Fig. 4. Equivalent circuit for deriving the transfer function of (a) $V_{\mathrm{X}} / V_{n, R S}$, and (b) $V_{\mathrm{Y}} / V_{n, R L 1}$.

As the frequency increases, the noise analysis becomes more complicated. The parasitic capacitances modify the impedance at various nodes and may cause incomplete noise cancellation at high frequencies. To derive the high-frequency NF, we temporarily neglect the noise of $M_{1}$ and define two transfer functions (Fig. 4) as follows:

$$
\begin{aligned}
& H_{0}(s)=\frac{V X}{V_{n, R S}}=\frac{1}{2} \frac{1}{\frac{L_{0} C_{0}}{2} s^{2}+\left(\frac{C_{0} R_{S}}{2}+\frac{L_{0}}{2 R_{S}}\right) s+1} \\
& H_{1}(s)=\frac{V_{Y}}{V_{n, R L 1}}=\frac{1}{L_{1} C_{1} s^{2}+C_{1} R_{L 1} s+1}
\end{aligned}
$$

where $C_{0}$ and $C_{1}$ denote the lumped parasitic capacitances at nodes $X$ and $Y$ in Fig. 4(a) and (b), respectively. Assuming
$R_{S}=1 / g_{m 1}$, the power spectral density of the output noise current due to $R_{S}$ is described by

$$
\begin{aligned}
S_{R S}(s)= & 4 k T R_{S}\left|g_{m 3}+g_{m 2} \frac{Z_{L 1}}{R_{S}}\right|^{2} \\
& \times\left|\frac{1 /\left(s C_{0}+1 / R_{S}\right)}{R_{S}+s L_{0}+1 /\left(s C_{0}+1 / R_{S}\right)}\right|^{2} \\
= & 4 k T R_{S}\left|g_{m 3}+g_{m 2} \frac{R_{L 1}}{R_{S}}\left(1+\frac{s L_{1}}{R_{L 1}}\right) H_{1}(s)\right|^{2} \\
& \times\left|H_{0}(s)\right|^{2}
\end{aligned}
$$

where $Z_{L 1}$ represents the impedance at node $Y$ in Fig. 4(b). The noise factor $F$ is given by

$$
F=1+F_{R L 1}+F_{M 2}+F_{M 3}
$$


where $F_{R L 1}, F_{M 2}$, and $F_{M 3}$ are derived as follows based on (4) and (10)-(12):

$$
\begin{aligned}
F_{R L 1}= & \frac{4 k T R_{L 1} g_{m 2}^{2}\left|H_{1}(s)\right|^{2}}{S_{R S}(s)} \\
= & \frac{R_{S}}{R_{L 1}}\left|\frac{1}{\left[1+s L_{1} / R_{L 1}+1 / H_{1}(s)\right] H_{0}(s)}\right|^{2} \\
F_{M 2}= & \frac{4 k T g_{m 2} \gamma / \alpha}{S_{R S}(s)} \\
= & \frac{R_{S}}{R_{L 1}} \frac{\gamma}{\alpha} \frac{1}{g_{m 2} R_{L 1}} \\
& \times\left|\frac{1}{\left[1+\left(1+s L_{1} / R_{L 1}\right) H_{1}(s)\right] H_{0}(s)}\right|^{2} \\
F_{M 3}= & \frac{4 k T g_{m 3} \gamma / \alpha}{S_{R S}(s)} \\
= & \frac{\gamma}{\alpha} \frac{1}{g_{m 3} R_{S}} \\
& \times\left|\frac{1}{\left[1+\left(1+s L_{1} / R_{L 1}\right) H_{1}(s)\right] H_{0}(s)}\right|^{2} .
\end{aligned}
$$

Note that each of (14)-(16) is composed of a low-frequency term and a frequency-dependent term. As $s \rightarrow 0$, (14)-(16) are reduced to (6)-(8), respectively. By substituting (10) and (11) into (14), the frequency response of $F_{R L 1}$ depends on the design parameters. This is because the resultant numerator and denominator of (14) are both second-order polynomials. On the other hand, the magnitude of $F_{M 2}$ and $F_{M 3}$ is expected to increase at high frequencies because the denominators of (15) and (16) are low-pass functions. The above analysis helps us understand the influence of each noise source on the overall NF. For example, the high-frequency NF may be more susceptive to the noise from $M_{2}$ and $M_{3}$. These observations lead to the design methodology of the LNA, which is presented in the following section.

\section{Design Methodology}

\section{A. Design of Input Common-Gate Stage}

By inspecting (9), the value of $R_{L 1}$ should be maximized to minimize the noise from $R_{L 1}$ and $M_{2}$. Therefore, a smaller size of $M_{1}$ is desirable in order to obtain a flat gain response up to $10 \mathrm{GHz}$. Considering the $50 \Omega$ matching condition, the width and the bias current of $M_{1}$ are chosen to be $30 \mu \mathrm{m}$ and $2.5 \mathrm{~mA}$, respectively. The maximum value of $R_{L 1}$ is limited by two constraints. First, increasing $R_{L 1}$ causes the required shunt-peaking inductor, $L_{1}$, to increase rapidly ( $L_{1}$ is proportional to $R_{L 1}^{2}$ ) [16]. This not only lowers the inductor self-resonance frequency due to the larger parasitic capacitance but also degrades the efficiency of the shunt peaking technique. Second, the drain voltage of $M_{1}$ should be higher than $\left(V_{\mathrm{GS} 1}+V_{\mathrm{GS} 3}-V_{\mathrm{TH} 1}\right)$ to keep $M_{1}$ in saturation. The choice of $R_{L 1}$ thus highly depends on the overdrive voltages of $M_{1}$ and $M_{3}$. Though a higher overdrive voltage makes the $f_{\mathrm{T}}$ higher, it causes velocity saturation in a deep-submicron MOSFET and makes the noise performance worse [13], [16]. Considering $M_{3}$ may become one of the main noise contributors, its overdrive voltage is designed

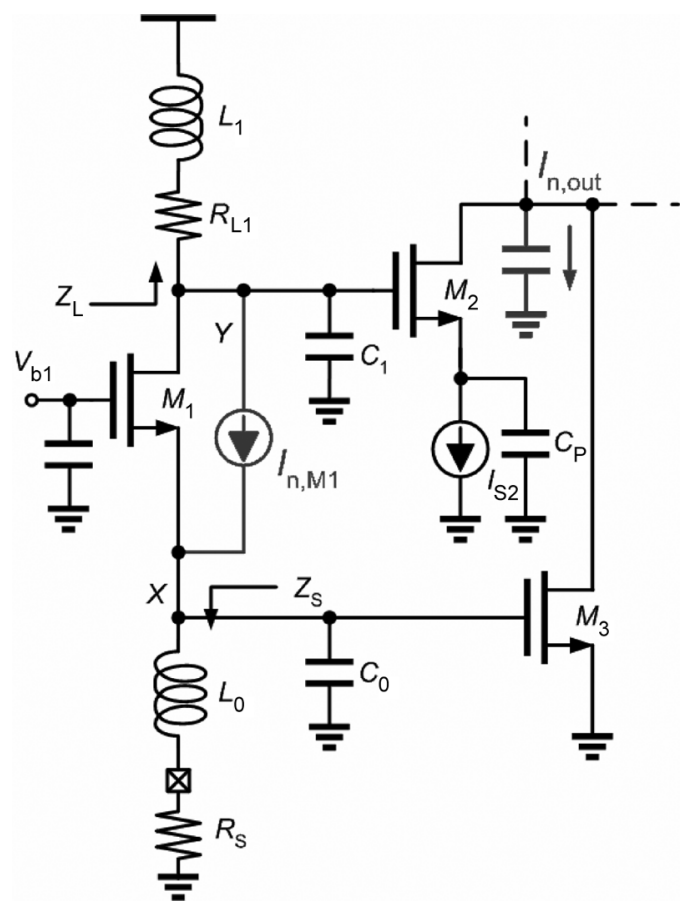

Fig. 5. Equivalent circuit for canceling the noise of $M_{1}$.

to be $150-200 \mathrm{mV}$. The above considerations result in the final choice of $R_{L 1}=180 \Omega$. This leads to a safe margin larger than $300 \mathrm{mV}$ for $M_{1}$ operating in saturation, which provides adequate linearity.

\section{B. Design of Noise-Canceling Stage}

The size and the bias of $M_{2}$ and $M_{3}$ must be chosen according to broadband noise cancellation as well as minimizing their noise. The equivalent circuit for canceling the noise of $M_{1}$ is depicted in Fig. 5. To examine the effect of noise cancellation, the output is ac-shorted to ground. The noise current, $I_{n, \text { out }}$ can be derived as

$$
\begin{aligned}
I_{n, \text { out }}= & \left(g_{m 2} Z_{L 1}-g_{m 3} Z_{S}\right) \frac{I_{n, M 1}}{1+g_{m 1} Z_{S}} \\
= & \left(g_{m 2} R_{L 1} \frac{1+s L_{1} / R_{L 1}}{L_{1} C_{1} s^{2}+C_{1} R_{L 1} s+1}\right. \\
& \left.-g_{m 3} R_{S} \frac{1+s L_{0} / R_{S}}{L_{0} C_{0} s^{2}+C_{0} R_{S} s+1}\right) \\
& \times \frac{I_{n, M 1}}{1+g_{m 1} Z_{S}}
\end{aligned}
$$

where $C_{0}$ and $C_{1}$ denote the lumped parasitic capacitances at nodes $X$ and $Y$, respectively. From (4) and (17), it is seen that the only way for frequency-independent noise cancellation is

$$
\frac{L_{1}}{L_{0}}=\frac{R_{L 1}}{R_{S}}=\frac{C_{0}}{C_{1}}
$$

Note that it is not impossible to obtain $\left(L_{1} / L_{0}\right)=\left(R_{L 1} / R_{S}\right)$ in typical IC design, since the values of inductors and resistors are primarily determined by the layout geometry and hence the lithography in the fabrication process. However, the parasitic capacitances $C_{0}$ and $C_{1}$ are dependent on bias condition and quite 
sensitive to process variation. Tight control of them is thus difficult. For a given bias current, a larger size of $M_{3}$ results in a higher $g_{m 3}$, reducing the last term in (9) and hence the overall NF. The maximum tolerable size of $M_{3}$ is specified by the input matching at high frequencies. Inductor $L_{0}$ and parasitic capacitance $C_{0}$ form an $L C$ ladder structure. When terminated with $1 / g_{m 1}=R_{S}$, it provides an input impedance equal to $\sqrt{L_{0} / C_{0}}$ with a cut-off frequency of $1 / \pi \sqrt{L_{0} C_{0}}$. As the frequency increases to $30 \%-40 \%$ of the cut-off frequency, the impedance begins to deviate [16]. It is thus desirable to design $L_{0}$ and $C_{0}$ with a high cut-off frequency. The inductor $L_{0}$ is chosen to be $0.7 \mathrm{nH}$, and the width of $M_{3}$ is limited to $120 \mu \mathrm{m}$ accordingly. With the value of $L_{0}$ determined, inductor $L_{1}$ is chosen to be $2.52 \mathrm{nH}$ according to (18). The size of $M_{2}$ must be designed considering the bandwidth at the output of the first stage. To obtain a flat gain response up to $10 \mathrm{GHz}, M_{2}$ is designed to be $30 \mu \mathrm{m}$ wide, which is one-fourth of $M_{3}$. The resultant $C_{0}$ and $C_{1}$ cannot exactly satisfy (18) since the size of $M_{2}$ and $M_{3}$ is tied to other constraints, such as bandwidth and input matching, respectively. Though the frequency-independent noise cancellation is hard to achieve, by inspecting (17), we can still find the optimized ratio of $g_{m 3}$ to $g_{m 2}$ that yields the widest frequency range of noise cancellation.

Assuming $\left(g_{m 3} R_{S} / g_{m 2} R_{L 1}\right)=1+\varepsilon(0<\varepsilon \ll 1)$, $\left(L_{1} / L_{0}\right)=\left(R_{L 1} / R_{S}\right)$, and $R_{S}=\left(1 / g_{m 1}\right)$, (17) can be rearranged as shown in (19) at the bottom of the page. By observing the transfer function, there are two zeros related to the parameter $\varepsilon$ and have greatest influence on the effect of noise cancellation. Given a total power consumption of $20 \mathrm{~mW}$, the bias currents of $M_{2}$ and $M_{3}$ are altered to find the optimized value of $\varepsilon$.

Consider the solutions of the following equation, which correspond to the zeros in (19):

$$
s^{2}\left[(1-\varepsilon) L_{0} C_{0}-L_{1} C_{1}\right]+s\left[(1-\varepsilon) C_{0} R_{S}-C_{1} R_{L 1}\right]-\varepsilon=0
$$

By substituting the estimated $C_{0}=280 \mathrm{fF}, C_{1}=90 \mathrm{fF}$, and other design parameters into (20), the behaviors of the two zeros with increasing $\varepsilon$ are illustrated in Fig. 6. With a small $\varepsilon$, the two zeros are real and different, while for a larger $\varepsilon$, they move toward each other and then separate along the $\omega=\omega_{0}$ axis. The value of $\varepsilon$ corresponding to $\omega=\omega_{0}$ is found to be 0.052 by solving the following equation:

$$
\left[(1-\varepsilon) C_{0} R_{S}-C_{1} R_{L 1}\right]^{2}+4 \varepsilon\left[(1-\varepsilon) L_{0} C_{0}-L_{1} C_{1}\right]=0
$$

while the frequency $f_{0}=\omega_{0} / 2 \pi$ is found to be $5.7 \mathrm{GHz}$. The above derivations suggest that for a small but finite $\varepsilon(0<\varepsilon<$

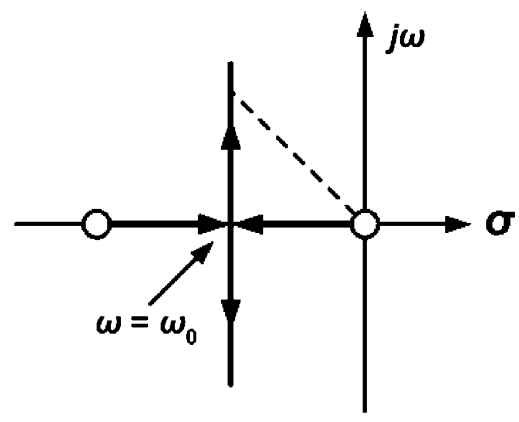

Fig. 6. Behaviors of the two roots of (20) with increasing $\varepsilon$.

0.052), the noise cannot be properly cancelled at the higher band. In this case, the two zeros are separated and at least one falls below $6 \mathrm{GHz}$, which causes $I_{n \text {,out }} / I_{n, M 1}$ to rise and degrades the in-band noise cancellation. On the other hand, for a too large $\varepsilon(\varepsilon \gg 0.052)$, the noise cannot be eliminated at the lower band because $I_{n, \text { out }} / I_{n, M 1}$ is proportional to $\varepsilon g_{m 3} R_{S}$ [see (19)] at low frequencies. In addition, the noise will deteriorate rapidly at high frequencies due to the two complex zeros. Fig. 7 depicts the simulated $I_{n \text {,out }} / I_{n, M 1}$ versus frequency with different values of $\varepsilon$. Due to the capacitor, $C_{\mathrm{p}}$, in Fig. 5, which attenuates the signals below $1 \mathrm{GHz}, I_{n \text {,out }} / I_{n, M 1}$ is somewhat large at low frequencies. However, the trend coincides with the above derivations. It is thus desirable to choose the $\varepsilon$ that yields the widest frequency range of noise cancellation. With $\varepsilon=0.25$, which corresponds to $g_{m 3} / g_{m 2}=4.5$, the noise power of $M_{1}$ is attenuated by a factor larger than 10 from 3.5 to $8.5 \mathrm{GHz}$ $\left(I_{n, \text { out }} / I_{n, M 1} \leq 0.3\right)$.

\section{Effect of Noise Cancellation}

To further confirm the effect of noise cancellation and to perform a fair comparison, the case with $M_{3}$ turned OFF is simulated with the same power dissipation by the following steps. 1) $V_{b 3}$ is set to zero. 2) $V_{b 1}$ is adjusted to increase the bias current of $M_{1}$ (original $I_{\mathrm{D} 1}=2.5 \mathrm{~mA}$ ) until it operates at the edge of saturation region $\left(V_{\mathrm{GD}}=V_{\mathrm{TH}}\right)$. 3) The bias current of $M_{2}$ is increased until the total power dissipation is the same as the case with $M_{3}$ turned ON. Note that no other changes such as $W_{1}$ (the size of $M_{1}$ ) and $R_{L 1}$ can be made because the bandwidth at node $Y$ [in Fig. 4(b)] must be maintained in order to meet the fullband UWB specification. The noise contributed by $M_{1}, R_{L 1}$ and $M_{3}$ normalized to the noise contributed by $R_{S}$, i.e., $F_{M 1}, F_{R L 1}$, and $F_{M 3}$, respectively, are then simulated. As shown in Fig. 8, with the same power dissipation and a similar

$$
\begin{aligned}
\frac{I_{n, \text { out }}}{I_{n, M 1}} & =\frac{1}{1+g_{m 1} Z_{S}}\left(g_{m 2} Z_{L 1}-g_{m 3} Z_{S}\right) \\
& \approx \frac{g_{m 3} R_{S}}{2} \frac{\left\{s^{2}\left[(1-\varepsilon) L_{0} C_{0}-L_{1} C_{1}\right]+s\left[(1-\varepsilon) C_{0} R_{S}-C_{1} R_{L 1}\right]-\varepsilon\right\}\left(1+\frac{s L_{1}}{R_{L 1}}\right)}{\left(\frac{L_{0} C_{0}}{2} s^{2}+\frac{C_{0} R_{S}+L_{0} / R_{S}}{2}+1\right)\left(L_{1} C_{1} s^{2}+C_{1} R_{L 1} s+1\right)}
\end{aligned}
$$




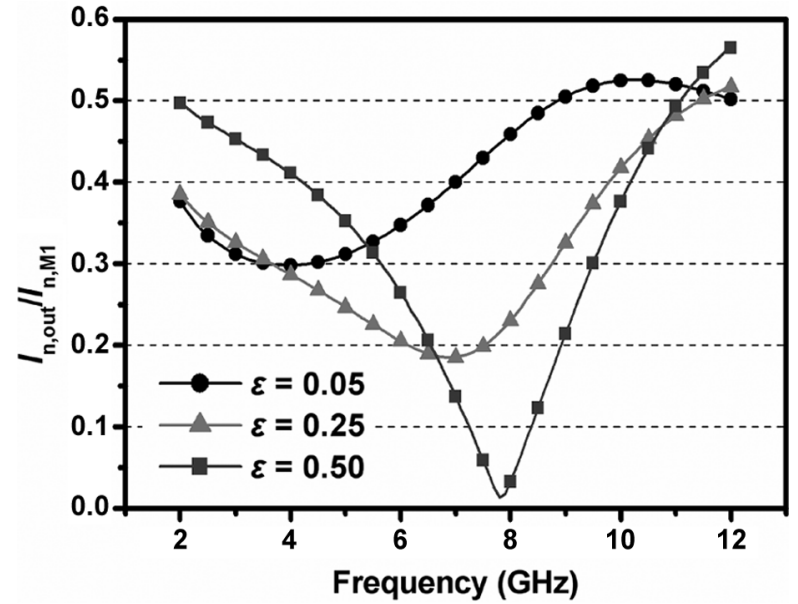

Fig. 7. Simulated $I_{n \text {,out }} / I_{n, M 1}$ versus frequency with different values of $\varepsilon$.

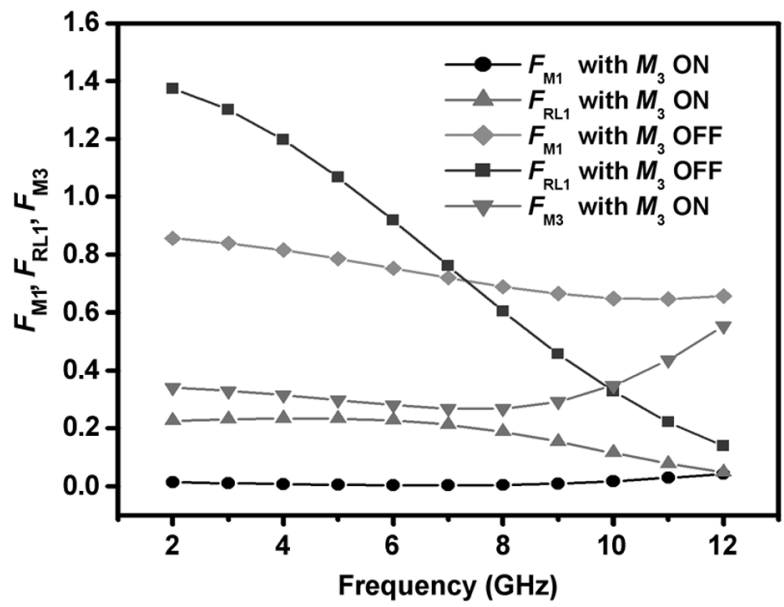

Fig. 8. Simulated $F_{M 1}, F_{R L 1}$, and $F_{M 3}$ with $M_{3}$ turned ON and OFF.

bandwidth, the noise factors contributed by $M_{1}$ and $R_{L 1}$ are reduced when $M_{3}$ is $\mathrm{ON}$, since the addition of $M_{3}$ not only cancels the noise (smaller $F_{M 1}$ ) but also amplifies the signal ${ }^{3}$ (smaller $\left.F_{R L 1}\right)$. Besides, the increase in the NF caused by adding $M_{3}$ is less than the decrease in the NF caused by canceling the noise of $M_{1}$, which is obtained by comparing $F_{M 3}$ with $F_{M 1}$ for the two cases over the entire UWB band.

\section{Final LNA Design}

With a deliberate mismatch between $g_{m 3} R_{S}$ and $g_{m 2} R_{L 1}$, the noise factor $F$ shows some dependence on $\varepsilon$ and can be re-derived as the following equations: 4

$$
F=1+F_{R L 1}+F_{M 2}+F_{M 3}
$$

${ }^{3}$ The result of lower $F_{R L 1}$ can also be explained by comparing (6) with (1). The factor of 4 diminishes due to the noise-canceling topology, which cannot be obtained by increasing the bias current of the original common-gate topology.

${ }^{4}$ Though (24)-(26) are derived as no frequency dependency, their high-frequency behaviors can be considered by following the same procedure in Section III. where $F_{R L 1}, F_{M 2}$, and $F_{M 3}$ are described by

$$
\begin{aligned}
F_{M 1} & =\frac{\left(g_{m 2} R_{L 1}-g_{m 3} R_{S}\right)^{2}\left[1 /\left(1+g_{m 1} R_{S}\right)^{2}\right] 4 k T g_{m 1} \gamma / \alpha}{k T R_{S}\left(g_{m 3}+g_{m 2} R_{L 1} / R_{S}\right)^{2}} \\
& \approx \frac{\gamma}{4 \alpha} \varepsilon^{2}(1+\varepsilon) \\
F_{R L 1} & =\frac{4 k T R_{L 1} g_{m 2}^{2}}{k T R_{S}\left(g_{m 3}+g_{m 2} R_{L 1} / R_{S}\right)^{2}} \\
& =\frac{R_{S}}{R_{L 1}} \frac{1}{(1+\varepsilon / 2)^{2}} \\
& \approx \frac{R_{S}}{R_{L 1}}(1-\varepsilon) \\
F_{M 2} & =\frac{4 k T g_{m 2} \gamma / \alpha}{k T R_{S}\left(g_{m 3}+g_{m 2} R_{L 1} / R_{S}\right)^{2}} \\
& =\frac{R_{S}}{g_{m 2} R_{L 1}^{2}} \frac{\gamma}{\alpha} \frac{1}{(1+\varepsilon / 2)^{2}} \\
& \approx \frac{R_{S}}{g_{m 2} R_{L 1}^{2}} \frac{\gamma}{\alpha}(1-\varepsilon) \\
F_{M 3} & =\frac{4 k T g_{m 3} \gamma / \alpha}{k T R_{S}\left(g_{m 3}+g_{m 2} R_{L 1} / R_{S}\right)^{2}} \\
& =\frac{\gamma}{\alpha} \frac{1}{g_{m 3} R_{S}} \frac{(1+\varepsilon)^{2}}{(1+\varepsilon / 2)^{2}} \\
& \approx \frac{\gamma}{\alpha} \frac{1}{g_{m 3} R_{S}}(1+\varepsilon) .
\end{aligned}
$$

For $0<\varepsilon<1$, it is observed that the noises from $R_{L 1}$ and $M_{2}$ decrease with increasing $\varepsilon$, while those from $M_{1}$ and $M_{3}$ increase. Thus, the choice of $\varepsilon$ must consider the efficiency of noise cancellation as well as the overall NF. However, by carefully inspecting (22)-(26), the total NF is expected to change little for some range of $\varepsilon$. This is because the noise from $M_{1}$ is proportional to $\varepsilon^{2}$, while the noise from $R_{L 1}, M_{2}$, and $M_{3}$ will not change very much for $\varepsilon \ll 1$. Fig. 9 depicts the simulated NF of this LNA averaged from 3.1 to $10.6 \mathrm{GHz}$ with different values of $\varepsilon$. It reaches a minimum of $3.906 \mathrm{~dB}$ for $\varepsilon=0.15$ and remains quite flat for $\varepsilon$ ranging from 0.05 to 0.2 . Based on these observations, we complete the design by choosing $I_{\mathrm{D} 2}=1.8 \mathrm{~mA}$ and $I_{\mathrm{D} 3}=6.5 \mathrm{~mA}$ to obtain $\varepsilon=0.15$. The flat behavior of average NF versus $\varepsilon$ makes this design not sensitive to process variation. As shown in Fig. 10, the simulated NF is also compared to the case with $M_{3}$ turned OFF. The NF is reduced by at least $2 \mathrm{~dB}$ with the same power dissipation and a similar bandwidth. Fig. 11 depicts the simulated noise contribution of $M_{1}$, $R_{L 1}, M_{2}$, and $M_{3}$ to the total output noise. Here the noise of $M_{\mathrm{x}}$ represents the channel thermal noise only. The output noise is dominated by $R_{L 1}$ and $M_{3}$ at low frequencies, while it is primarily dominated by $M_{3}$ as the frequency approaches $10 \mathrm{GHz}$.

The simulated $S$ parameters of this LNA with $M_{3}$ turned ON and OFF, respectively, are shown in Fig. 12. Again, the comparison is performed with the same power dissipation and a similar bandwidth. The power gain is $10-11 \mathrm{~dB}$ from 3.1 to $10.6 \mathrm{GHz}$, which is $3-4 \mathrm{~dB}$ larger compared to the case with $M_{3}$ OFF. It is worth noting that without $M_{3}$, the gain of the common-gate topology can only be increased by increasing $R_{L 1}$ because of the input matching constraint. However, increasing $R_{L 1}$ inevitably decreases the bandwidth at the output of the first stage. In other words, the increase in the gain and the decrease 


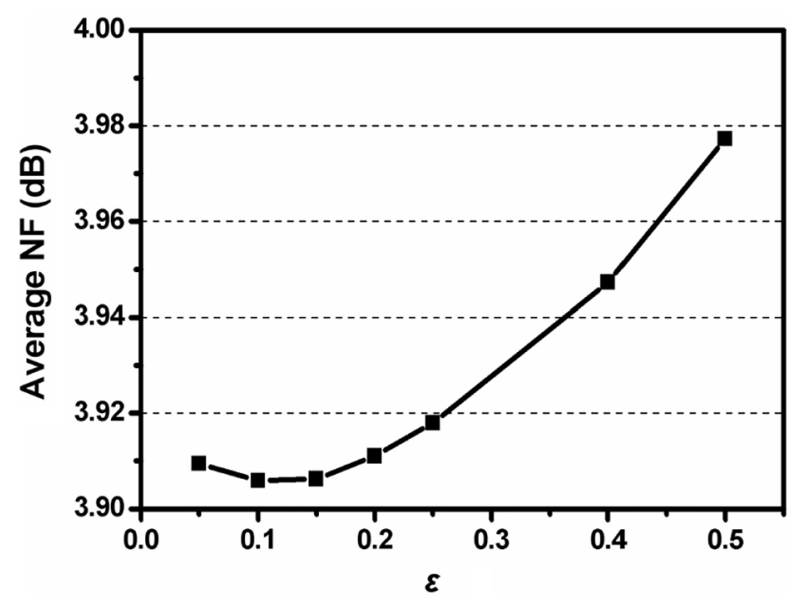

Fig. 9. Simulated NF averaged from 3.1 to $10.6 \mathrm{GHz}$ with different values of $\varepsilon$.

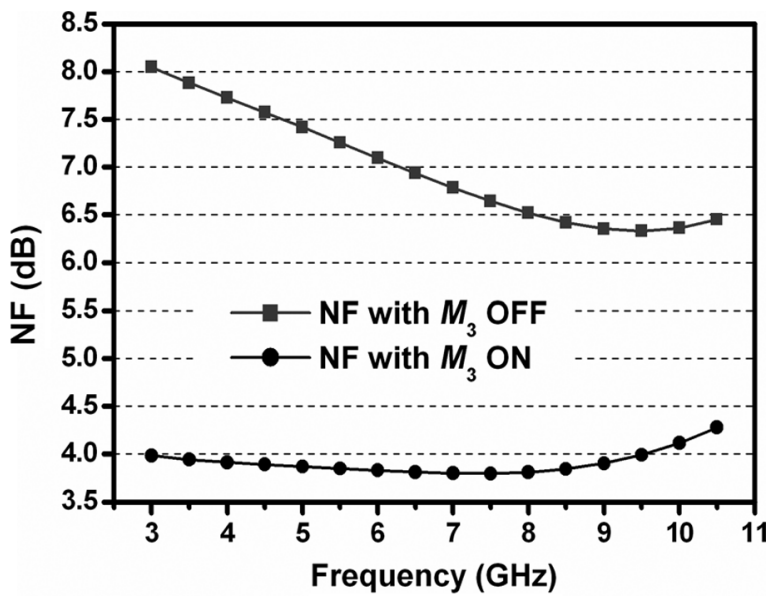

Fig. 10. Simulated NF of this LNA with $M_{3}$ turned ON and OFF.

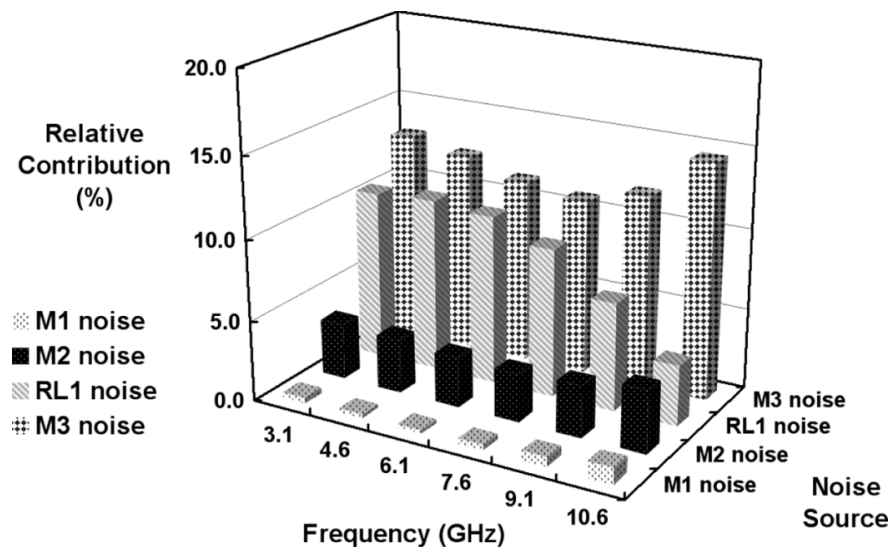

Fig. 11. Simulated noise contribution of $M_{1}, R_{L 1}, M_{2}$, and $M_{3}$ to the total output noise.

in the NF are the fundamental advantages of the noise-canceling topology. The output buffer consumes $5 \mathrm{~mA}$ and features $4 \mathrm{~dB}$ loss due to the $50 \Omega$ on-chip termination. ${ }^{5}$ In an integrated receiver, the LNA can directly drive I/Q mixers and the voltage

${ }^{5} \mathrm{~A}$ common source buffer instead of a source follower is used here to emulate a more realistic loading of mixers on the LNA core. The width of $M_{4}$ is $80 \mu \mathrm{m}$, which is greater than typical loading of I/Q mixers operating at 3-10-GHz.

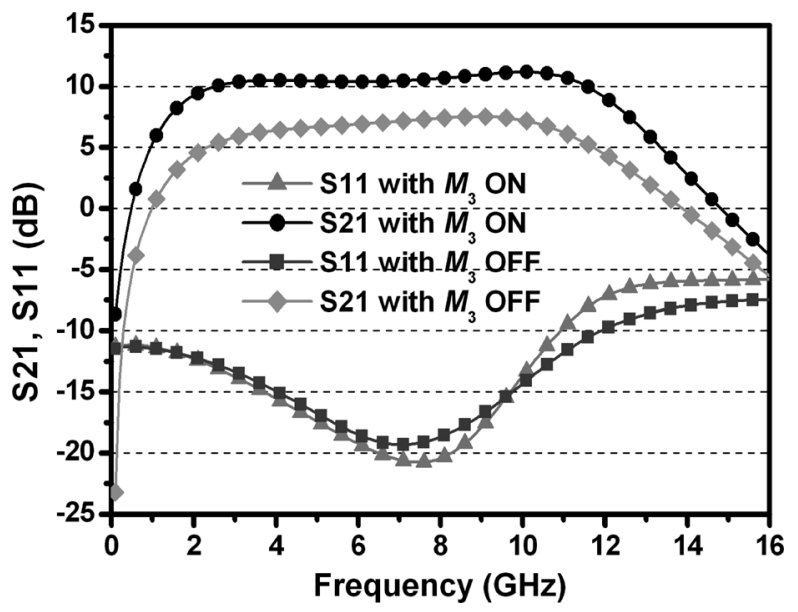

Fig. 12. Simulated $S$ parameters of this LNA with $M_{3}$ turned $\mathrm{ON}$ and OFF.

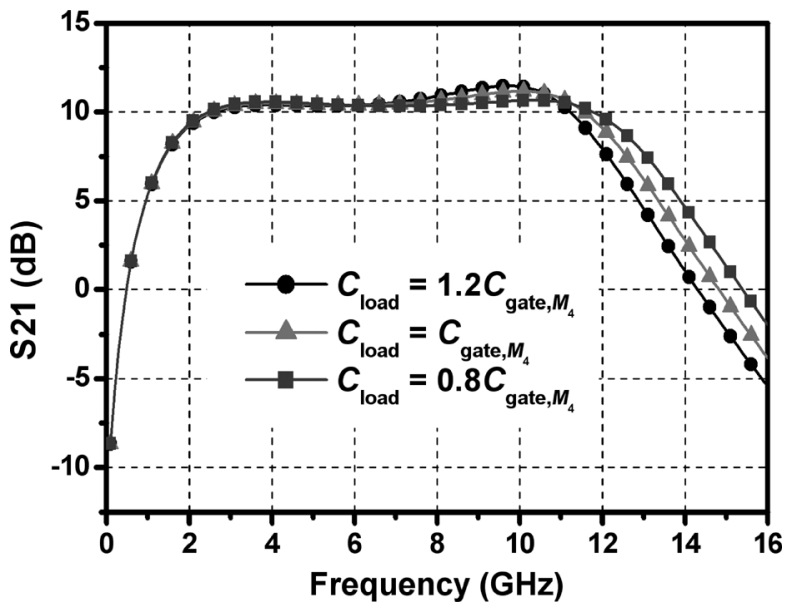

Fig. 13. Simulated $S_{21}$ with different capacitance loaded on the core.

gain is expected to be $4 \mathrm{~dB}$ higher. To verify that the bandwidth will not change very much due to the variation of the capacitance loaded on the core, the $S_{21}$ is simulated with the gate capacitance of $M_{4}$ varied by $\pm 20 \%$. As shown in Fig. 13, a larger $C_{\text {load }}$ causes a slightly greater in-band peaking but a smaller bandwidth because the resonance point moves to lower frequency. Without any modification of the core design, the gain at $10.6 \mathrm{GHz}$ varies by only $0.4 \mathrm{~dB}$ for the three cases. The design values of this LNA are summarized in Table I, where the core consumes $20 \mathrm{~mW}$ from a 1.8-V supply.

\section{EXPERIMENTAL RESULTS}

A prototype of this LNA has been fabricated in a $0.18-\mu \mathrm{m}$ CMOS process. The die micrograph is shown in Fig. 14. The total area including the output buffer and the pads is $0.9 \times$ $0.65 \mathrm{~mm}^{2}$. The measurements have been carried out on wafer.

$S$ parameters and NF are measured using an ATN NP5B measurement system. Fig. 15 shows the measured power gain and output return loss. Measured power gain achieves a maximum of $9.7 \mathrm{~dB}$ at $4.2 \mathrm{GHz}$, and remains $1-\mathrm{dB}$ flatness from 2.4 to $9.4 \mathrm{GHz}$. The lower and upper $-3 \mathrm{~dB}$ bandwidth are $1.2 \mathrm{GHz}$ and $11.9 \mathrm{GHz}$, respectively. The measured gain is less flat than 
TABLE I

DESIGN VALUES OF THE LNA

\begin{tabular}{|c|c|c|c|}
\hline \multirow{2}{*}{ Transistor size } & $(W / L)_{1,2}$ & $(W / L)_{3}$ & $(W / L)_{4}$ \\
\cline { 2 - 4 } & $30 / 0.18$ & $120 / 0.18$ & $80 / 0.18$ \\
\hline \multirow{3}{*}{ Bias current } & $I_{\mathrm{D} 1}$ & $I_{\mathrm{D} 2}$ & $I_{\mathrm{D} 3}$ \\
\cline { 2 - 4 } & $2.5 \mathrm{~mA}$ & $1.8 \mathrm{~mA}$ & $6.5 \mathrm{~mA}$ \\
\hline \multirow{2}{*}{ Resistor value } & $R_{\mathrm{L} 1}$ & $R_{\mathrm{L} 2}$ & $R_{\mathrm{L} 3}$ \\
\cline { 2 - 4 } & $180 \Omega$ & $90 \Omega$ & $50 \Omega$ \\
\hline \multirow{2}{*}{ Inductor value } & $L_{0,4}$ & $L_{1}$ & $L_{2,3}$ \\
\cline { 2 - 4 } & $0.7 \mathrm{nH}$ & $2.52 \mathrm{nH}$ & $1.18 \mathrm{nH}$ \\
\hline
\end{tabular}

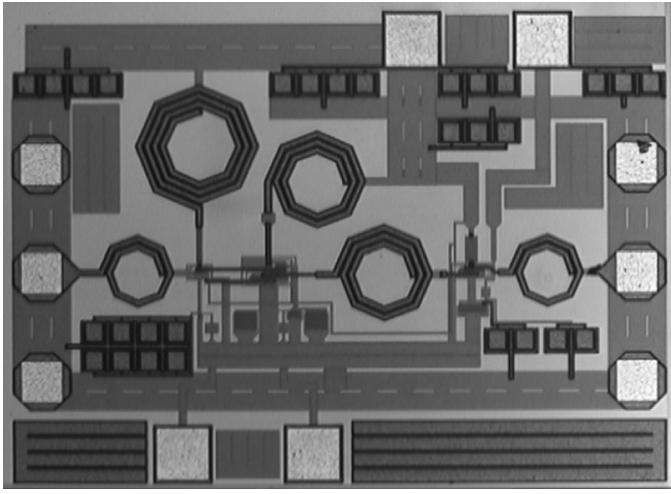

Fig. 14. Die micrograph of the LNA.

the simulated one. It is believed that the inductor $L_{3}$ is over-estimated in simulation, which causes the real gain to exhibit a lower bandwidth and a smoother out-of-band roll-off. The measured output return loss is larger than $10 \mathrm{~dB}$ up to $14 \mathrm{GHz}$. It shows different trend at low frequencies compared with the simulation. This may attribute to process variation of $R_{L 3}$ and the limited frequency range $(1-18 \mathrm{GHz})$ of tuners used for noise measurement in this test plan. Fig. 16 shows the measured input return loss and reverse isolation: $S_{11}$ is below $-10 \mathrm{~dB}$ up to $14 \mathrm{GHz}$, while $S_{12}$ is below $-35 \mathrm{~dB}$ across the band of interest.

The measured NF is illustrated in Fig. 17. Simulated NFs with $\gamma$ equal to $2 / 3$ and $4 / 3$ are put together for comparisons. The measurement shows a NF of $4.5-5.1 \mathrm{~dB}$ over $3.1-10.6 \mathrm{GHz}$, and the average value is $4.7 \mathrm{~dB}$. The measured $\mathrm{NF}$ is $0.7-1 \mathrm{~dB}$ larger compared to the simulation with $\gamma=2 / 3$. However, it is close to the simulated NF with $\gamma=4 / 3$. In addition, the trend of the measured NF agrees with that of the simulated result over the entire UWB band. This confirms the effect of broadband noise cancellation as well as the proposed design methodology.

Fig. 18 shows the measured IIP3 at $6 \mathrm{GHz}$. Applying two tones with $1-\mathrm{MHz}$ spacing, the measured IIP3 is $-6.2 \mathrm{dBm}$, while the input referred $\mathrm{P}_{1 \mathrm{~dB}}$ is found to be $-16 \mathrm{dBm}$. The measured IIP3 versus frequency is shown in Fig. 19. In the frequency range of 3-6 GHz, the IIP3 ranges from -4.9 to $-6.2 \mathrm{dBm}$.

Fig. 19 also shows the measured IIP2 versus frequency. It is measured by applying two tones with $200-\mathrm{MHz}$ spacing. The IIP2 is higher than $+9.7 \mathrm{dBm}$ in the $3-6-\mathrm{GHz}$ range. This is primarily because the capacitor $C_{\mathrm{hp}}$ features low impedance to

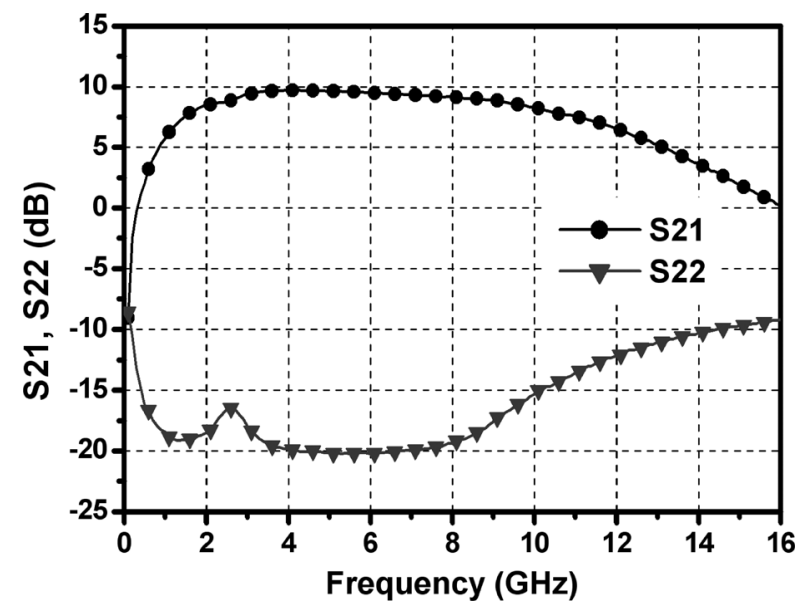

Fig. 15. Measured power gain and output return loss.

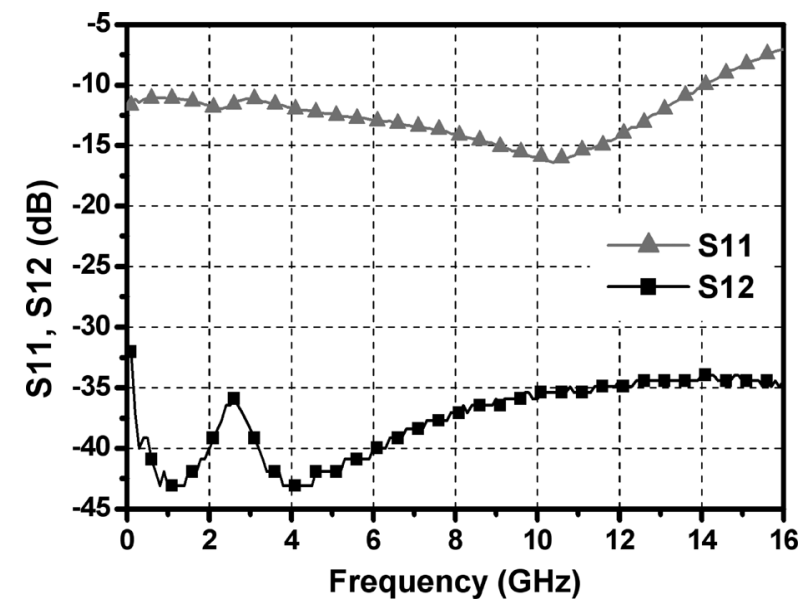

Fig. 16. Measured input return loss and reverse isolation.

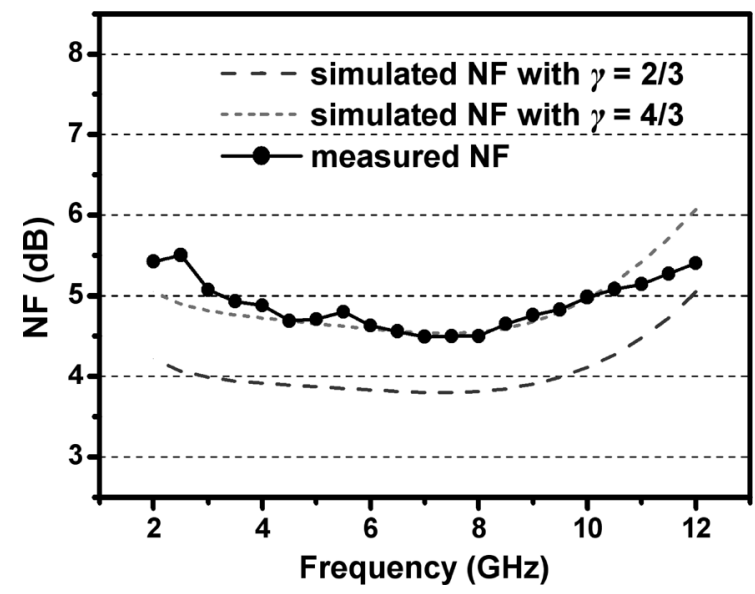

Fig. 17. Measured NF compared with simulations.

ground at the signal frequency and high impedance at low frequencies [23], [24]. The IM2 tone is thus attenuated considerably relative to the main tone, resulting in a higher IIP2. Since the output buffer can be reconfigured as a single-balanced mixer by replacing $R_{L 3}$ with a switching quad, the measured IIP3 and IIP2 can be treated as the overall effects of the LNA core and the transcondutor of the mixer. 
TABLE II

Measured Performance Compared With Prior Published Works

\begin{tabular}{c|c|c|c|c|c|c|c|c}
\hline \hline Ref. & $\begin{array}{c}\text { CMOS } \\
\text { Technology }\end{array}$ & $\begin{array}{c}S_{11} \\
(\mathrm{~dB})\end{array}$ & $\begin{array}{c}G_{\max } \\
(\mathrm{dB})\end{array}$ & $\begin{array}{c}\mathrm{BW}_{3-\mathrm{dB}} \\
(\mathrm{GHz})\end{array}$ & $\begin{array}{c}\mathrm{NF}^{+} \\
(\mathrm{dB})\end{array}$ & $\begin{array}{c}\mathrm{IIP3} \\
(\mathrm{dBm})\end{array}$ & $\begin{array}{c}\text { Power } \\
(\mathrm{mW})\end{array}$ & $\begin{array}{c}\text { Area } \\
\left(\mathrm{mm}^{2}\right)\end{array}$ \\
\hline \hline This Work & $\mathbf{0 . 1 8}-\boldsymbol{\mu \mathrm { m }}$ & $<-\mathbf{1 1}$ & $\mathbf{9 . 7}$ & $\mathbf{1 . 2}-\mathbf{1 1 . 9}$ & $\mathbf{4 . 5}-\mathbf{5 . 1}$ & $-\mathbf{6 . 2}$ & $\mathbf{2 0}$ & $\mathbf{0 . 5 9}$ \\
\hline$[4]$ & $0.18-\mu \mathrm{m}$ & $<-11$ & 11.5 & $0.5-14$ & $3.4-5.4$ & +10 & $52^{*}$ & 1.6 \\
\hline$[5]$ & $0.18-\mu \mathrm{m}$ & $<-16$ & 8.6 & $0.03-7$ & $4.2-6.2$ & +1.8 & $9^{*}$ & 1.16 \\
\hline$[8]$ & $0.13-\mu \mathrm{m}$ & $<-9$ & 16 & 5.9 & $4.7-5.7$ & - & $38^{*}$ & 0.24 \\
\hline$[9] \mathrm{STD}$ & $0.18-\mu \mathrm{m}$ & $<-9.9$ & 9.3 & $2.3-9.2$ & $4-8$ & -6.7 & 9 & 1.1 \\
\hline$[9] \mathrm{TW}$ & $0.18-\mu \mathrm{m}$ & $<-9.4$ & 10.4 & $2.4-9.5$ & $4.2-8$ & -8.8 & 9 & 1.1 \\
\hline$[10]$ & $0.25-\mu \mathrm{m}$ & $<-8$ & 13.7 & $0.02-1.6$ & $1.9-2.4$ & 0 & $35^{*}$ & 0.075 \\
\hline$[21]$ & $0.18-\mu \mathrm{m}$ & $<-9$ & 9.8 & $2-4.6$ & $2.3-5.2$ & -7 & 12.6 & 0.9 \\
\hline$[22]$ & $0.13-\mu \mathrm{m}$ & $<-10$ & 19 & $0.1-6.5$ & $3-4.2$ & +1 & 12 & - \\
\hline \hline
\end{tabular}

: This work specifies the NF in the entire UWB band, while others specify the NF over their 3-dB bandwidth.

*: Total power including buffer, ours is $29 \mathrm{~mW}$

Table II Measured performance compared with prior published works.

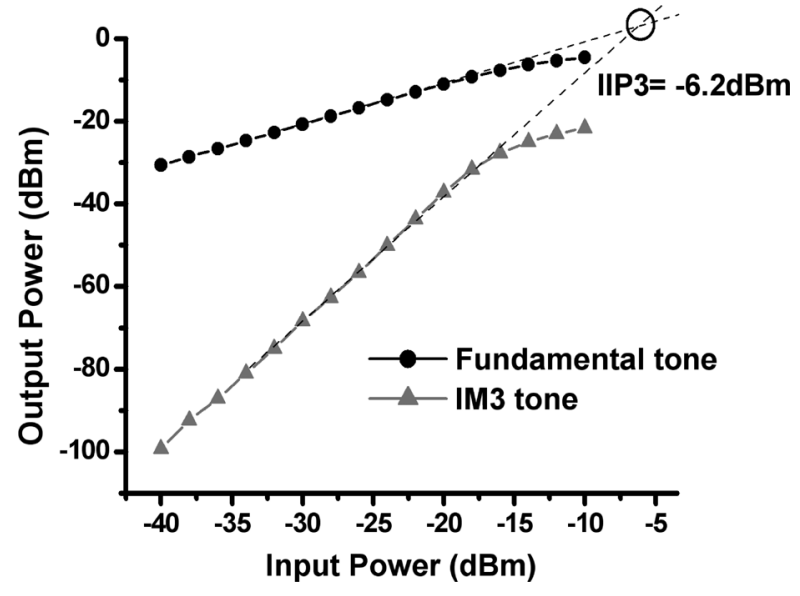

Fig. 18. Measured IIP3 at $6 \mathrm{GHz}$.

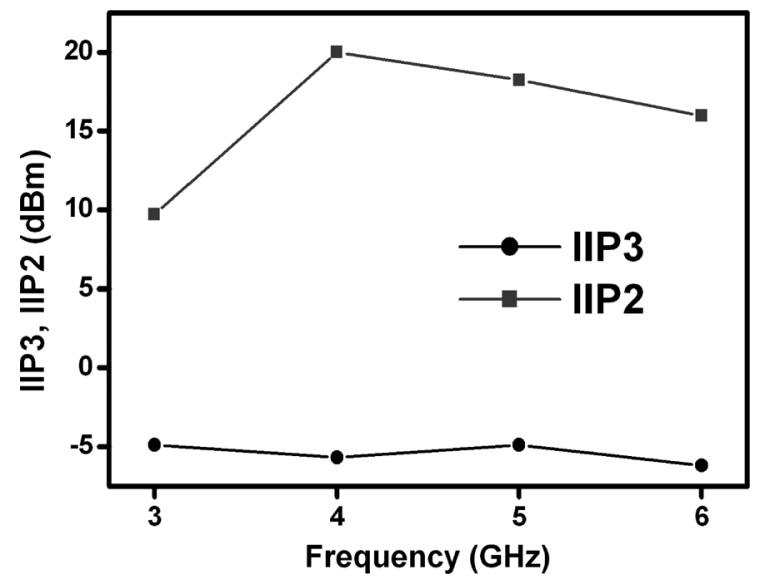

Fig. 19. Measured IIP3 and IIP2 versus frequency.
The measured performance of this LNA is summarized in Table II. Other previously published wideband LNAs, especially those for UWB systems, are included for comparisons. The proposed circuit benefits from high bandwidth, low average NF over the entire UWB band, and small die area with moderate power consumption.

\section{CONCLUSION}

A broadband noise-canceling LNA for 3.1-10.6-GHz UWB receivers has been demonstrated in a $0.18-\mu \mathrm{m}$ CMOS technology. By utilizing inductive peaking techniques and the proposed design methodology, the effective operating frequency of noise cancellation can be brought to the desired UWB band, while other performance such as matching and bandwidth is separately optimized. The additional noise from circuits performing noise cancellation is minimized through a detailed noise analysis and a systematic design approach. By reducing the number of high- $Q$ inductors required in a filter-based topology, the proposed circuit occupies an area of only $0.59 \mathrm{~mm}^{2}$, which facilitates a low cost design. Experimental results show a flat NF of $4.5-5.1 \mathrm{~dB}$ over the entire UWB band, a peak gain of $9.7 \mathrm{~dB}$ with $-3 \mathrm{~dB}$ bandwidth from 1.2 to $11.9 \mathrm{GHz}$, and an IIP3 of $-6.2 \mathrm{dBm}$, while consuming $20 \mathrm{~mW}$ from a $1.8-\mathrm{V}$ supply.

\section{ACKNOWLEDGMENT}

The authors would like to thank MediaTek Inc. and NSC for support of this work, Chip Implementation Center (CIC) for chip fabrication, and Radio Frequency Technology Center (RFTC) for measurement support. 


\section{REFERENCES}

[1] S. Stroh, "Ultra-wideband: multimedia unplugged," IEEE Spectrum, vol. 40, no. 9, pp. 23-27, Sep. 2003.

[2] A. Batra et al., "Multi-band OFDM physical layer proposal," IEEE 802.15-03/267r5, Jul. 2003.

[3] G. R. Aiello and G. D. Rogerson, "Ultra-wideband wireless systems," IEEE Microwave Mag., vol. 4, no. 2, pp. 36-47, Jun. 2003.

[4] R.-C. Liu, C.-S. Lin, K.-L. Deng, and H. Wang, "A 0.5-14-GHz 10.6-dB CMOS cascode distributed amplifier," in Symp. VLSI Circuits Dig. Tech. Papers, Jun. 2003, pp. 139-140.

[5] F. Zhang and P. Kinget, "Low power programmable-gain CMOS distributed LNA for ultra-wideband applications," in Symp. VLSI Circuits Dig. Tech. Papers, Jun. 2005, pp. 78-81.

[6] H. Knapp, D. Zoschg, T. Meister, K. Aufinger, S. Boguth, and L. Treitinger, " $15 \mathrm{GHz}$ wideband amplifier with $2.8 \mathrm{~dB}$ noise figure in SiGe bipolar technology," in Proc. IEEE Radio Frequency Integrated Circuits Symp., Jun. 2003, pp. 287-290.

[7] S. Andersson, C. Svensson, and O. Drugge, "Wideband LNA for a multistandard wireless receiver in $0.18 \mu \mathrm{m}$ process," in Proc. Eur. SolidState Circuits Conf. (ESSCIRC), Sep. 2003, pp. 655-658.

[8] R. Gharpurey, "A broadband low-noise front-end amplifier for ultra wideband in $0.13 \mu \mathrm{m}$ CMOS," in Proc. IEEE Custom Integrated Circuits Conf., Oct. 2004, pp. 605-608.

[9] A. Bevilacqua and A. M. Niknejad, "An ultra-wideband CMOS LNA for 3.1 to $10.6 \mathrm{GHz}$ wireless receivers," in IEEE ISSCC Dig. Tech. Papers, Feb. 2004, pp. 382-383.

[10] F. Bruccoleri, E. A. M. Klumperink, and B. Nauta, "Noise canceling in wideband CMOS LNAs," in IEEE ISSCC Dig. Tech. Papers, Feb. 2002, pp. 406-407.

[11] — "Wide-band CMOS low-noise amplifier exploiting thermal noise canceling," IEEE J. Solid-State Circuits, vol. 39, no. 2, pp. 275-282, Feb. 2004

[12] C.-F. Liao and S.-I. Liu, "A broadband noise-canceling CMOS LNA for 3.1-10.6-GHz UWB receiver," in Proc. IEEE Custom Integrated Circuits Conf., Sep. 2005, pp. 161-164.

[13] D. K. Shaeffer and T. H. Lee, "A 1.5-V, 1.5-GHz CMOS low noise amplifier," IEEE J. Solid-State Circuits, vol. 32, no. 5, pp. 745-759, May 1997.

[14] A. J. Scholten, H. J. Tromp, L. F. Tiemeijer, R. van Langevelde, R. J. Havens, P. W. H. De Vreede, R. F. M. Roes, P. H. Woerlee, A. H. Montree, and D. B. M. Klaassen, "Accurate thermal noise modeling for deep-submicron CMOS," in IEDM Tech. Dig., Dec. 1999, pp. 155-158.

[15] A. J. Scholten, L. F. Tiemeijer, R. Van Langevelde, R. J. Havens, A. T. A. Zegers-van Duijnhoven, and V. C. Venezia, "Noise modeling for RF CMOS circuit simulation," IEEE Trans. Electron Devices, vol. 50, no. 3, pp. 618-632, Mar. 2003

[16] T. H. Lee, The Design of CMOS Radio-Frequency Integrated Circuits, 1st ed. New York: Cambridge Univ. Press, 1998.

[17] W. Zhuo, S. Embabi, J. Pineda de Gyvez, and E. Sanchez-Sinencio, "Using capacitive cross-coupling technique in RF low noise amplifiers and down-conversion mixer design," in Proc. Eur. Solid-State Circuits Conf. (ESSCIRC), Sep. 2000, pp. 77-80.

[18] B. Razavi, T. Aytur, F.-R. Yang, R.-H. Yan, H.-C. Kang, C.-C. Hsu, and C.-C. Lee, "A $0.13 \mu \mathrm{m}$ CMOS UWB transceiver," in IEEE ISSCC Dig. Tech. Papers, Feb. 2005, pp. 216-217.

[19] S. S. Mohan, M. D. M. Hershenson, S. P. Boyd, and T. H. Lee, "Bandwidth extension in CMOS with optimized on-chip inductors," IEEE J. Solid-State Circuits, vol. 35, no. 3, pp. 346-355, Mar. 2000.
[20] S. Galal and B. Razavi, " $40 \mathrm{~Gb} / \mathrm{s}$ amplifier and ESD protection circuit in $0.18 \mu \mathrm{m}$ CMOS technology," in IEEE ISSCC Dig. Tech. Papers, Feb. 2004, pp. 480-481.

[21] C.-W. Kim, M.-S. Kang, P. T. Anh, H.-T. Kim, and S.-G. Lee, "An ultra-wideband CMOS low noise amplifier for 3-5-GHz UWB system," IEEE J. Solid-State Circuits, vol. 40, no. 2, pp. 544-547, Feb. 2005.

[22] S. Chehrazi, A. Mirzaei, R. Bagheri, and A. A. Abidi, "A 6.5 GHz wideband CMOS low noise amplifier for multi-band use," in Proc. IEEE Custom Integrated Circuits Conf., Sep. 2005, pp. 801-804.

[23] D. Manstretta, M. Brandolini, and F. Svelto, "Second-order intermodulation mechanisms in CMOS downconverters," IEEE J. Solid-State Circuits, vol. 38, no. 3, pp. 394-406, Mar. 2003.

[24] M. Brandolini, P. Rossi, D. Sanzogni, and F. Svelto, "A CMOS direct down-converter with $+78 \mathrm{dBm}$ minimum IIP2 for $3 \mathrm{G}$ cell-phones," in IEEE ISSCC Dig. Tech. Papers, Feb. 2005, pp. 320-321.

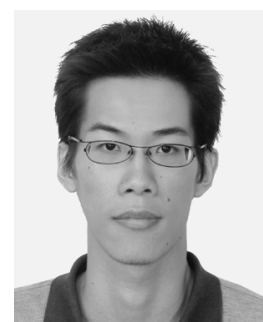

Chih-Fan Liao (S'05) was born in Taipei, Taiwan, R.O.C., in 1981. He received the B.S. degree in electric engineering from National Taiwan University, Taipei, in 2003. He is currently working toward the $\mathrm{Ph} . \mathrm{D}$. degree at the Graduate Institute of Electronics Engineering, National Taiwan University.

His research topic focuses on broadband CMOS circuit design for high-speed data communication systems.

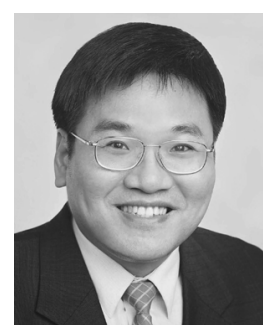

Shen-Iuan Liu (S'88-M'93-SM'03) was born in Keelung, Taiwan, R.O.C., 1965. He received the B.S. and Ph.D. degrees in electrical engineering from National Taiwan University (NTU), Taipei, in 1987 and 1991, respectively.

During 1991-1993, he served as a Second Lieutenant in the Chinese Air Force. During 1991-1994, he was an Associate Professor in the Department of Electronic Engineering of National Taiwan Institute of Technology. He joined in the Department of Electrical Engineering, NTU, Taipei, in 1994 and he has been a Professor since 1998. His research interests are in analog and digital integrated circuits and systems.

Dr. Liu has served as Chair of IEEE SSCS Taipei Chapter from 2004. He has served as General Chair of the 15th VLSI Design/CAD Symposium, Taiwan, 2004, and Program Co-chair of the Fourth IEEE Asia-Pacific Conference on Advanced System Integrated Circuits, Japan, 2004. He was the recipient of the Engineering Paper Award from the Chinese Institute of Engineers, 2003 the Young Professor Teaching Award from MXIC Inc., the Research Achievement Award from NTU, and the Outstanding Research Award from National Science Council, 2004. He served as a technical program committee member for A-SSCC and ISSCC, 2005-2006. In 2006, he became an Associate Editor for the IEEE JOURNAL OF SOLID-STATE CIRCUITS and an Associate Editor for IEEE TRANSACTIONS ON CIRCUITS AND SYSTEMS-II: EXPRESS BRIEFS. He is a member of IEICE. 